\title{
Emotion Recognition and Understanding Using EEG Data in A Brain-Inspired Spiking Neural Network Architecture
}

\author{
Wael Alzhrani ${ }^{1,2}$, Maryam Doborjeh ${ }^{1 *}$, Zohreh Doborjeh ${ }^{3,4}$, Nikola Kasabov ${ }^{1,5}$ \\ w.alzhrani@.tvtc.gov.sa ; mgholami@aut.c.nz; zohreh.doborieh@auckland.ac.nz; nkasabov@aut.ac.nz
}

\begin{abstract}
${ }^{1}$ School of Engineering, Computing and Mathematical Sciences, Auckland University of Technology (AUT), Auckland, New Zealand.
${ }^{2}$ General Directorate Curricula, Technical and Vocational Training Corporation (TVTC), Riyadh, Saudi Arabia

${ }^{3}$ Audiology Department, School of Population Health, Faculty of Medical and Health Sciences, The University of Auckland, New Zealand.

${ }^{4}$ Centre for Brain Research, The University of Auckland, New Zealand

${ }^{5}$ George Moore Chair of Data Analytics, Ulster University, UK
\end{abstract}

\begin{abstract}
This paper is in the scope of emotion recognition by employing a recurrent spiking neural network (BI-SNN) architecture for modelling, mapping, learning, classifying, visualising, and understanding of spatio-temporal Electroencephalogram (EEG) data related to different emotional states. It further explores, develops, and applies a methodology based on the NeuCube BI-SNN, that includes methods for EEG data encoding, data mapping into a 3-dimensional BI-SNN model, unsupervised learning using spike-timing dependent plasticity (STDP) rule, spike-driven supervised learning, output classification, network analysis, and model visualisation and interpretation. The research conducted to model different emotional subtypes through mapping both space (brain regions) and time (brain dynamics) components of EEG brain data into SNN architecture. Here, a benchmark EEG dataset was used to design an empirical study that consisted of different experiments for classification of emotions. The obtained accuracy of $94.83 \%$ for EEG classification of four types of emotions was superior when compared with traditional machine learning techniques. The BI-SNN models not only detected the brain activity patterns related to positive and negative emotions with a high accuracy, but also revealed new knowledge about the brain areas activated in relation to different emotions. The research confirmed that neural activation increased in the frontal sites of brain $(F 7, F 3, A F 4)$ associated with positive emotions, while in the case of the negative emotions, connectivity strength was concentrated in the frontal (F4, AF3, F7, F8) and parietal sites of the brain (P7, P8).
\end{abstract}

Keywords- EEG, Emotion recognition, Classification, Spiking Neural Networks.

\section{INTRODUCTION}

$\mathbf{E}$ motion plays a vital role in human's daily communication. Precise identification of emotional subtypes can offer a better understanding of human mental states including anxiety and depression [1]. Emotional expressions are correlated with different physiological aspects [4] that may also be influenced by external stimuli integrating acoustic and visual incentives [2,3]. There are several instances of improved applications through emotion recognition techniques, such as social network communications [4, 5], and patients' communication who have difficulties in expressing their emotions (Autism) [6,7].

Emotion recognition problem has typically been conducted through the investigation of human expressions using multi-modal procedures, including physiology, text, audio, or video [8]. Several studies incorporated automated emotion recognition with respect to facial expressions, speech, and body gestures [9] by developing various techniques such as Bayesian networks [10], Gaussian Mixture models [11] and Hidden Markov Models [12]. Some of popular machine learning techniques for emotion recognition are Support Vector Machines (SVM), Maximum Entropy, and Naive Bayes. Deep learning techniques such as Convolutional Neural Network (CNN), Extreme Learning Machine (ELM), and Long Short-term Memory (LSTM) have also shown great promise in the identification of emotion types [13] in realworld applications including computer vision, speech recognition, and Natural Language Processing (NLP) [14]. Hitherto, emotion recognition through deep leaning methods were conducted by using multiple data inputs such as Electroencephalogram (EEG) brainwaves [15], audio-visual data [16], facial expression, and body gestures [1].

EEG records spatio-temporal brain data (STBD) with a considerable temporal resolution that expose brain cortical activities in millisecond precision. Thus far, EEG has been effectively used for assessing brain activity with specific performance in comprehension of cognitive patterns related to neurological and mental issues [17-21]. In [22], an emotion recognition experiment was conducted with respect to the impacts of audio and visual stimuli on 25 individuals' EEG brainwaves. The accuracy of emotion identification was $78 \%$ for valence film clips and $82 \%$ for an arousal state. In [23], $87 \%$ accuracy of emotion recognition was achieved using an individual-channel Brain Computer Interface (BCI) system and $\mathrm{H} 2 \mathrm{O}$ deep learning neural circuitry, which was based on a multi-layer feedforward artificial neural network. In [24], emotion recognition was developed through modelling of combined facial movements and EEG signals, when fine participants were instructed to watch specific film clips for stimulating 5 emotions in their minds. The EEG features space was reduced via principal component analysis (PCA), while SVM method was applied to categorize the selected vectors within various classes of emotion. One of the key attributes of these emotion recognition techniques is to achieve a reasonably high accuracy of emotion classification. While the aforementioned methods achieved good classification accuracy, none of them offered model interpretation that can help to reveal and understand the brain activities related to 
different emotions. Emotion recognition using EEG and neuro-computational models can offer an enhanced level of accuracy and interpretability in BCI systems, that effectively improves the interaction between humans and machines [25]. However, the interpretation of different types of emotion and the underpinnings of cognitive functions have not yet been investigated in depth.

In recent years, Neuroinformatics has emerged in the development of neuro-computational techniques to create models of different types of spatio-temporal brain data collected from neural activities relating to several functions including perception, vision, hearing, regulation, speech recognition, and memory [26-30] .

In this paper, a brain-inspired Spiking Neural Network (BI-SNN) NeuCube architecture [31-34] is used for modelling, visualising, learning, and classification of EEG data recorded during an emotion-related task that stimulates nine emotional subtypes: excitement, amusement, sadness, fear, anger, disgust, happiness, calmness, and surprise. The paper extends the proposed earlier methods for using NeuCube on EEG data [7, 19, 26, 27, 35-46] with a new method and experiments for emotion recognition. The BISNN structure is a 3-dimensional (3D) space of spiking neurons that spatially map a brain template. The $4^{\text {th }}$ dimension is time during which the temporal information in EEG timeseries is learned in the BI-SNN model to investigate the patterns in brain data. We applied BI-SNN models to study the EEG data related to positive emotions (calmness and happiness) and negative emotions (fear and anger). Then, different subtypes of emotions are classified. The methodology also includes the interpretation of the BI-SNN models that illustrate the activated brain areas corresponding to different types of emotions.

The structure of this paper is presented as follows: Section II explains the structure and methodology of the NeuCube BISNN architecture and the proposed method for using NeuCube for the task of emotion recognition. Section III reports the results of EEG data classification of different emotional states, and BI-SNN interpretation, as well as a comparative analysis using traditional machine learning tools. Section IV contains the conclusion and future work.

\section{METHODS AND MATERIALS}

This research is conducted to classify different emotional subtypes, but more importantly to comprehend the patterns in the brain that are associated with emotional states by applying a brain-inspired SNN architecture. Spiking Neural Networks (SNNs) have been deployed in various tasks such as picture classification, pattern recognition and function approximation [34]. The brain-inspired structures execute the operations and various tasks of computation using the communications of spikes through a network of neurons, which are linked across synapses [47-49]. The learning procedures of SNN result in forming connection weights between the neurons, the adjustment of which is achieved by a spike-interchange process. The weights modified in the synapses rely on parameters of learning and the precise time of spike emissions from pre-synaptic and post-synaptic neurons [50]. SNNs can be used for different learning methods (unsupervised, supervised learning) and can create different network architectures (such as feedforward and recurrent) [34].

In this study, we designed a recurrent BI-SNN architecture in NeuCube framework (available at https://kedri.aut.ac.nz/Rand-D-Systems/neucube) which is an evolving spatiotemporal data machine based on the SNN for modelling, learning, classification/regression, clustering, visualising and interpretation of spatio-temporal data. NeuCube SNN has been employed for modelling of various spatio-temporal dataset across different applications [18, 32]

Here, the framework of SNN emerged via a biologically pragmatic model of the brain that learns from input data by connecting large groups of spiking neurons [51, 52]. The SNN model and its computational architecture used in this research involve various algorithms that enable it to better learn, represent and analyse EEG brain data. As can be seen from Fig. 1, a NeuCube SNN system consists of the following five sub-modules/procedures [31, 34]:

- Input data encoding and mapping.

- Unsupervised learning in a 3D BI-SNN reservoir, called SNNcube.

- Supervised learning for classification/regression in an output evolving SNN classifier.

- Parameter optimisation.

- Model visualisation and interpretation.

Fig. 1(1) illustrates the EEG data measurement while participants were watching several emotional video clips [2]. Figs. 1, 2 and 3 demonstrate the procedure of employing SNN for encoding, mapping, learning, classifying, and visualising of EEG data. Figs. 1 and 2a depict encoding of EEG signals into spike trains using a threshold-based and representation method (TBR). Figs. 1 and $2 b$ show the mapping of EEG variables into a $3 \mathrm{D} \mathrm{SNN}$ reservoir with respect to neural coordinates $(x, y, z)$ as placed in the Talairach brain atlas [40]. Talairach template [53] was utilized in the neuronal coordinates as an essential aspect in the reservoir dimensions. The SNN initialisation was then performed using the SmallWorld $(S W)$ connectivity rule with radios=2.5. After the SNN mapping, the SNN model was trained using the unsupervised spike timing-dependent plasticity (STDP) learning [50]. The mentioned procedures are explained in the following sections.

\section{A. Encoding and Mapping of Input Data}

The input sub-module first encodes the input data (which, in this research is EEG) into spike trains to precisely represent significant changes in the brain signals over time. In this paper, we use a threshold-based representation (TBR) method [54] for EEG data encoding to spike trains. The created spike trains represent variations in the EEG signals that passed the threshold TBR. Then the data is spatially mapped into a brain structured SNN reservoir that is designed according to a topological information of brain regions in Talairach. An SNN model is scalable and compatible with different brain templates such as MNI [55], Talairach [53], or coordinates of individual brain data [29]. Then, input neurons are assigned in 


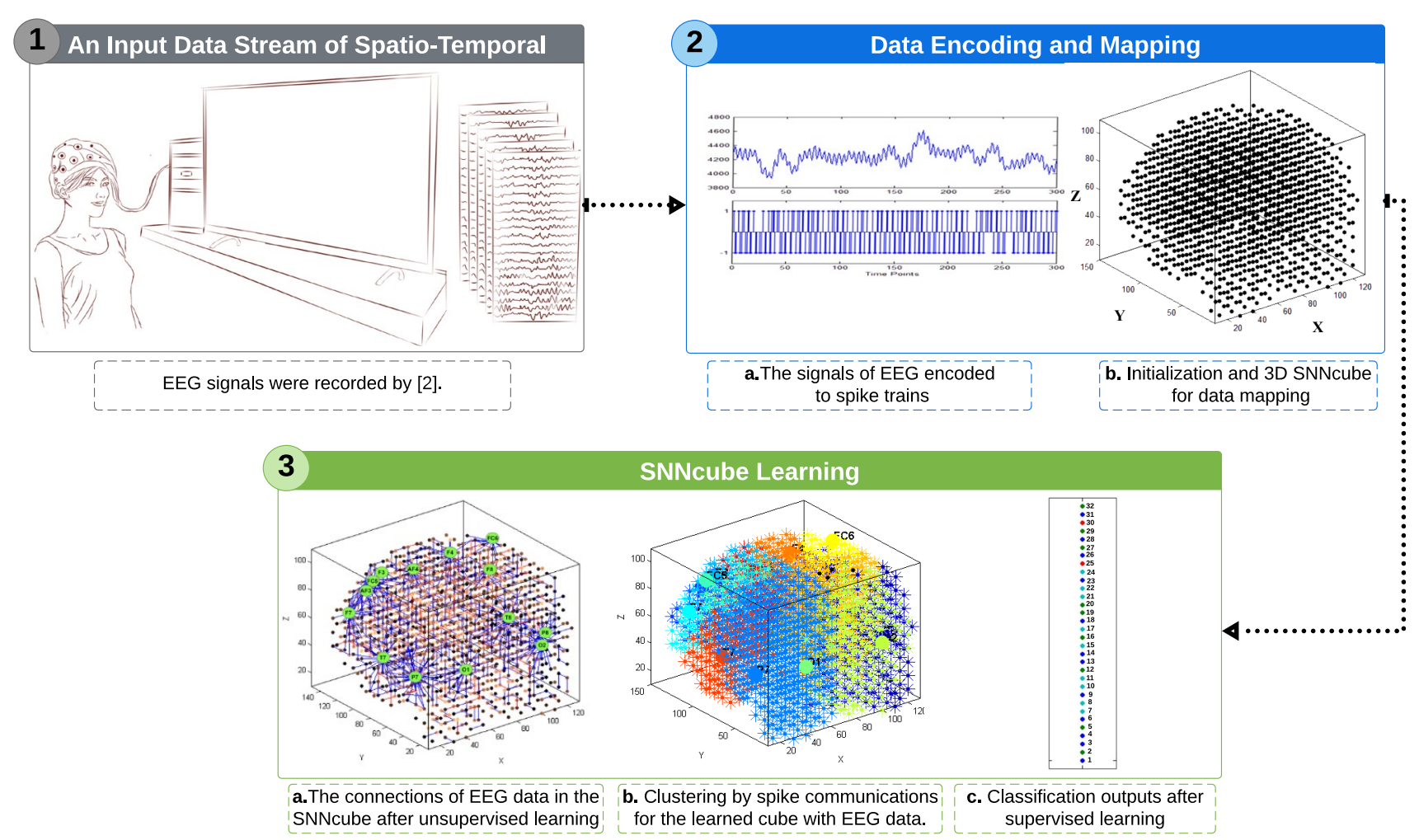

Fig. 1. The SNNcube architecture processes the input infortion as follows: encoding EEG data to spike trains; mapping to 3-dimensional SNNcube; unsupervised learning for EEG dataset; supervised learning and classification.

the SNN model to transfer the spike trains. Every input neuron has the same $(x, y, z)$ coordinate as positioned in a brain atlas [41]. Lastly, the neurons in the SNN are initially connected to each other using the Small-World (SW) connectivity rule [31, 56]. The initial neural connections will be modified during the learning process.

\section{B. SNN Learning Process using EEG Data}

The unsupervised spike timing-dependent plasticity (STDP) learning rule is employed to modify the SNN neural connections while streaming the training spatio-temporal EEG samples via the input neurons [31]. STDP learning rule is based on the Hebbian's theory [50], which distributes a steady stream of synaptic conductance with respect to the arrangement of receiving spikes, contributing to control of the spike possibilities over time [33].

\section{Supervised Learning}

This training stage is carried out utilising dynamic evolving SNN (deSNN) [57], where the same data series that are used for the unsupervised learning phase are deployed again to the SNN model, to train the output neurons of the classifier. All neurons in the SNNcube model are connected to each output neuron, and output neurons are created, or "evolved", to capture patterns of spatio-temporal activity in the SNN model related to different class labels. The activated sets of neurons will be used for classification/regression of the input data.

\section{Parameter Optimisation}

The proposed methodology in this paper contains different methods and parameters. Here we employed an exhaustive GRID search approach [58] with an objective function of reducing the testing error of the classification. Some of the main parameters that can be selected in the optimisation process are as follows:

- A self-adaptive bidirectional threshold for spike encoding. - Distance threshold that is employed for the neuron connectivity initialisation using small-world connectivity.

- STDP learning rate.

- Parameters of the deSNN classifier: mod (modulation factor) and drift (adjusting the connection weights in a positive or negative way depending on the spikes that followed the first spike at a synapse) [57].

\section{E. EEG Dataset Description}

We used the benchmark DREAMER EEG dataset [2] recorded from 23 healthy participants (14 Males, 9 Females) with an average age of 26.50 while watching four diverse film clips. There were 18 visual and audio clips, which have been evaluated effectively with respect to several emotions [59]. The EEG data were recorded using a wearable wireless headset with 14 channels (AF3, F7, F3, FC5, T7, P7, O1, O2, P8, T8, FC6, F4, F8, AF4), with sampling rate of $128 \mathrm{~Hz}$. The videos contained clips from numerous films to evoke a wide range of emotions (excitement, amusement, sadness, fear, anger, disgust, happiness, calmness, and surprise). The EEG data was recorded while the emotions of the participants were 
rated according to their sense of arousal, valence, and dominance.

Fig. 2 illustrates the analysis of two classes of EEG data (positive and negative emotions) using surface plots and signal-to-noise ratio (SNR) [60]. The SNR measures the power of each variable (EEG channel) for discriminating samples belonging to different classes in a classification task. It can be observed from Fig. 2(b) that the highest SNR values connected to variables 7, 1, and 5 (O1, AF3, T7 EEG channels) by $0.27,0.24,0.21$ values, respectively. This represents the best variables for discriminating and classifying EEG samples in the calmness and happiness classes (positive emotions). On the other hand, Fig. 2(d) demonstrates that variables T7, AF4, and P7 (with SNR values of $0.17,0.16$, 0.15 ) have greater ability to discriminate/classify EEG samples in the fear and anger classes (negative emotions).

\section{F. SNN-based Experimental Design}

The current research objectives are:

1. Classification of emotional subtypes through modelling of EEG data in a recurrent brain inspired SNN architecture.

2. Visualisation of the spatio-temporal relationships between the EEG channels in a 3D SNN reservoir for pattern recognition and understanding of the brain dynamics.

3. Comparing the results obtained via SNN models versus traditional machine-learning techniques (MLR, MLP, and RBF).

To this aim, we designed four experimental sessions (depicted in Fig. 3) as follows:

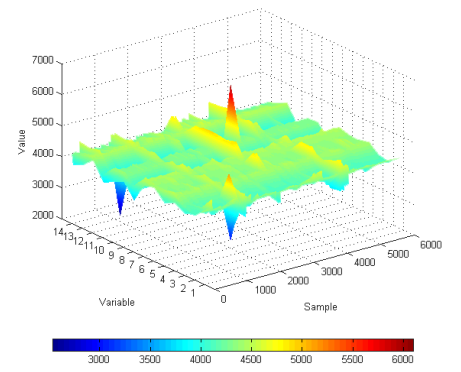

(a)

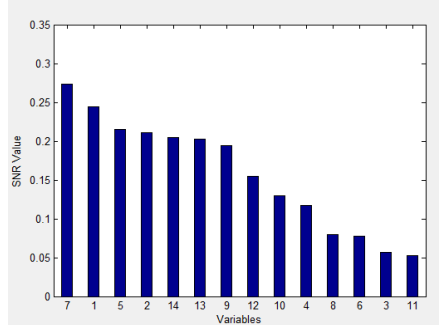

(c)

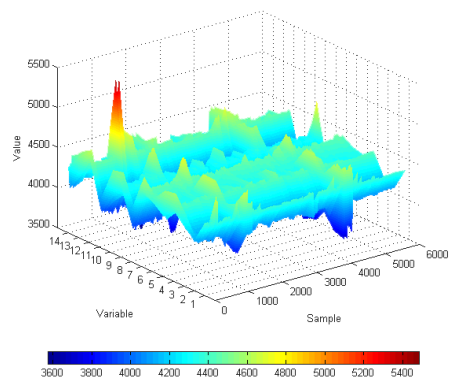

(b)

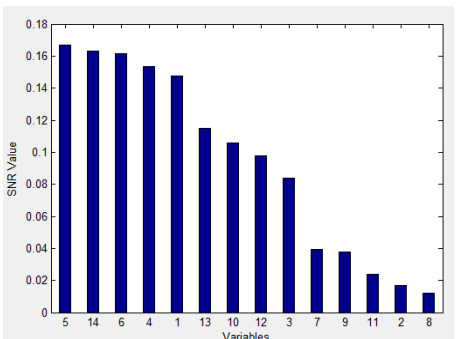

(d)

Fig. 2. EEG data analysis using surface plots (in a and b) and signal to noise ratio (in c and d) for positive and negative emotions correspondingly.

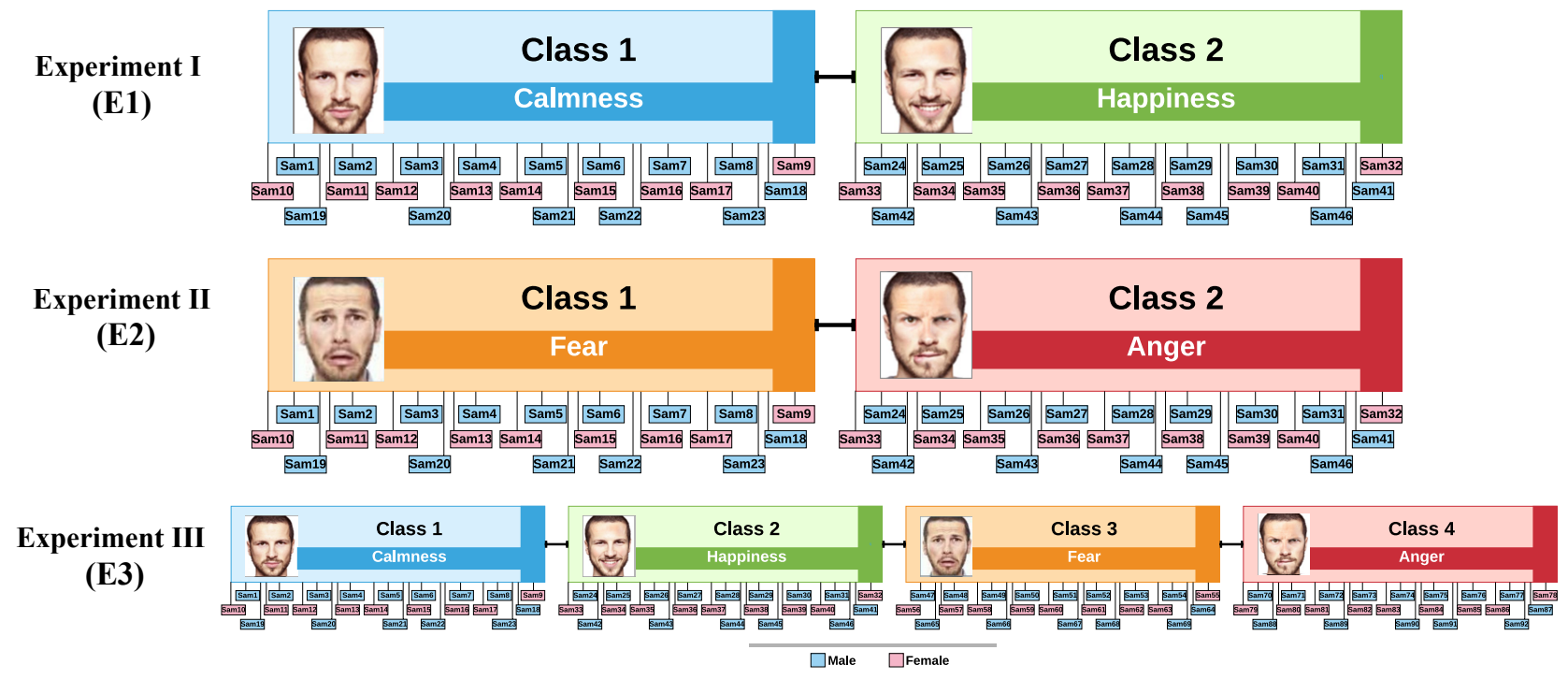

Fig. 3. The classification and samples of three datasets in NeuCube format. The images are from https://www.kingsnews.org 
Experiment 1 (E1): Unsupervised training of a NeuCube model on EEG data of calmness and happiness, referred as positive emotions (named E1). There are 46 EEG samples (23 spatio-temporal samples per class).

Experiment 2 (E2): Unsupervised learning of EEG data of fear and anger, referred as negative emotions (named E2). There are 46 EEG samples (23 samples per class).

Experiment 3 (E3): Unsupervised learning in a NeuCube model and supervised learning for classification of positive verses negative emotions.

Experiment 4 (E4): Classification of EEG data into the four types of emotions (calmness, fear, happiness, and anger) that cover both positive and negative emotions (named E3). There are 92 EEG samples (23 samples per class).

In all experiments, the EEG datasets have the same spatiotemporal dimension across all the samples (128 data time points representing one second measurement from 14 EEG electrodes).

\section{RESULTS}

In order to study and classify different emotional states, three SNN models were trained under unsupervised learning with EEG data related to experiments E1 to E4.

\section{A. Visualisation of the $3 D S N N$ Models Trained with EEG Data via STDP Learning}

Fig. 4 illustrates that the SNN models were trained with different EEG sample files (from E1, E2, and E3) through unsupervised STDP learning rule. Here, the visualised SNN models are computational models that can be studied and interpreted to better understand the EEG data and the underpinning brain functions. They captured the spatiotemporal associations between the EEG channels and the corresponding brain regions. The positive neural connections in the SNN models are displayed in blue lines, while negative connections are in red. The brighter colour of neurons represents a greater number of spikes was generated during the learning process, reflecting more activated brain regions.

The SNN models represent the differences between the EEG data related to different emotions. For instance, it can be seen from Fig. 4(a) and Fig. 4(b) that fear and anger emotions have elicited stronger SNN connectivity than calmness and happiness emotions. Furthermore, it can be observed from Fig. 4 that the activity of different regions of the brain revealed that there is an increase in the connectivity of SNN concerning the frontal (F7, F3, AF4), frontocentral (FC6), and parietal part $(\mathrm{P} 7, \mathrm{P} 8)$ in the samples of positive emotions. The intensity of this connectivity was focused on the frontal parts of the brain (F4, AF3, F7, F8), frontocentral (FC5, FC6), occipital areas $(\mathrm{O} 2)$ and parietal parts $(\mathrm{P} 7, \mathrm{P} 8)$ in the samples of negative emotions. More comprehensive activation occurred in the frontal areas of the brain for calmness and happiness emotional states, while the significant activation occurred over the frontal and parietal sites for anger and fear emotions.

\section{B. Network Analysis through Neuronal Clustering and Feature Intercation}

After the unsupervised learning process is completed, the spatio-temporal connections that were formed during STDP learning with EEG data in the trained SNN models can be further analysed. Here, we used clustering method to group the model's neurons with respect to their level of spike communication during the learning process. As shown in Fig. 5 , the input neurons are allocated to the cluster centres and labelled by the input variables (EEG channels). The rest of the neurons are then clustered by the centres with respect to the number of spikes that were received from each centre. Every neuron belongs to a cluster centre (EEG channel) that received the greatest number of spikes from that centre as compared to the other centres [51]. It can be seen from Fig. 5(a to c) that EEG datasets of experiments E1, E2, and E3 developed different clustering patterns in the trained SNN models.

Fig. 5(d to $\mathrm{f})$ illustrates an information route analysis traced by spike max gradient for the top three input neurons. Fig. 5(d) suggests that in E1 experiment, channels P7, T8, and $\mathrm{O} 2$ with neuron proportions of $21 \%, 19 \%$, and $16 \%$ respectively obtained the greatest spike gradient. Fig. 5(e) shows that channels $\mathrm{P} 7, \mathrm{P} 8$, and $\mathrm{T} 8$ with neuron percentage of $23 \%, 19 \%$, and $13 \%$ respectively had the greatest spike max gradient in E2 experiment. On the other hand, Fig. 5(f) illustrates that in E3 experiment the biggest spike max gradients were captured in channels $\mathrm{P} 7, \mathrm{~T} 8$, and AF4 with neuron proportion of $25 \%, 19 \%, 9 \%$ respectively.

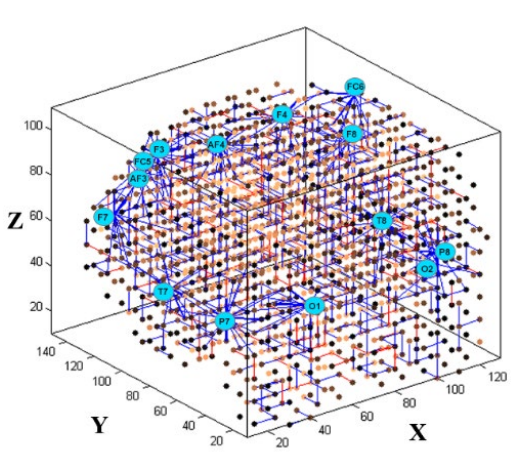

(a)

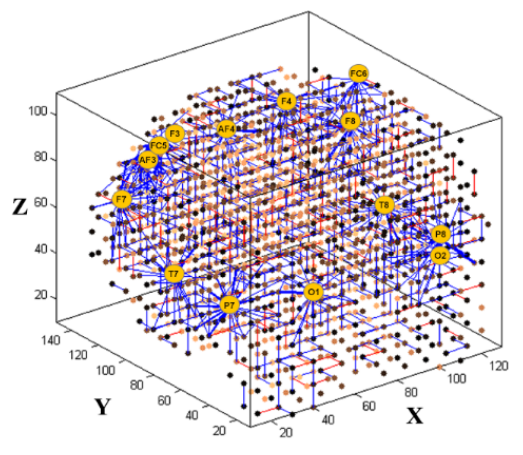

(b)

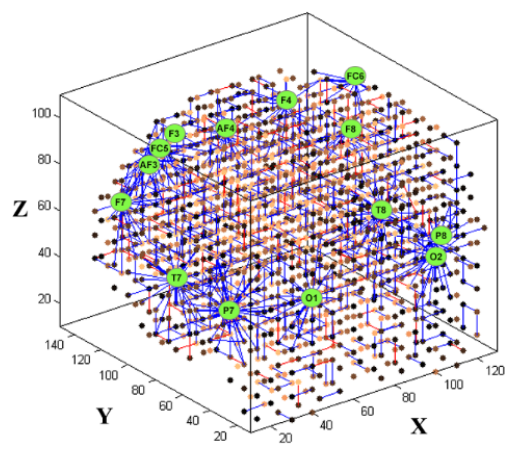

(c)

Fig. 4. SNN models connectivity after unsupervised STDP learning with three experimental EEG datasets. (a) SNN trained with 46 EEG samples of positive emotions, (b) SNN trained with 46 EEG samples of negative emotions, (c) SNN trained with 92 EEG samples of both positive and negative emotions. 
Positive Emotions (E1)

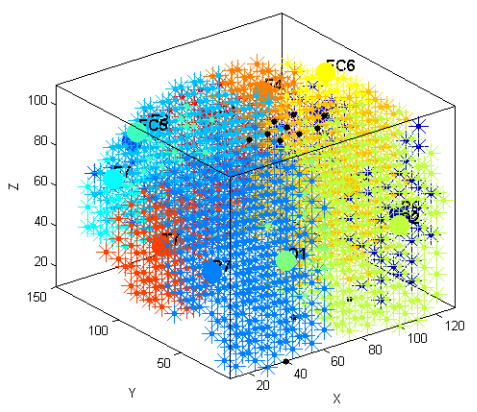

(a)

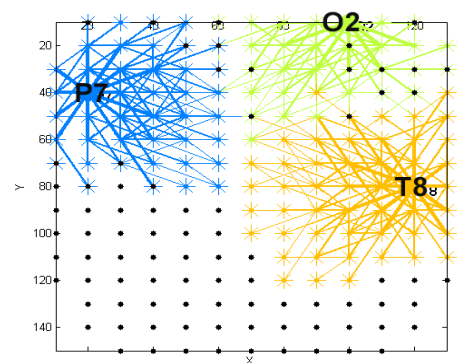

(d)

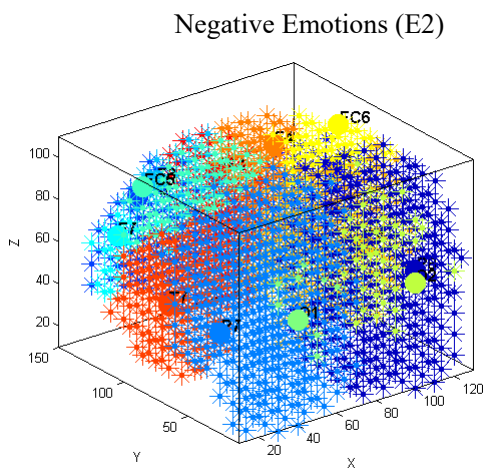

(b)

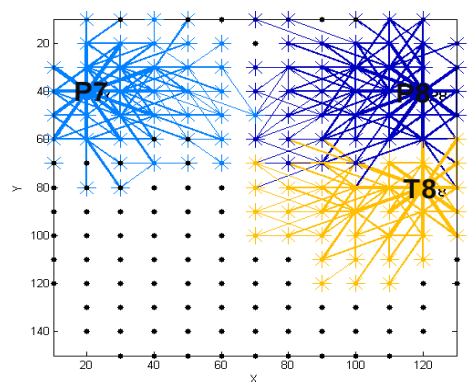

(e)
Both Emotions (E3)

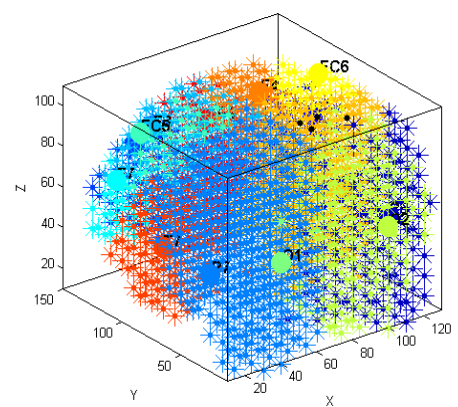

(c)

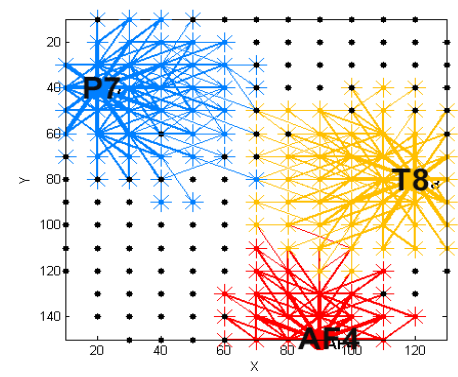

(f)

Fig. 5. Neural clustering by spike communications in the SNN models of EEG data related to positive emotions (in a), negative emotions (in b), and both EEG data from positive and negative emotions (in c). The spike max gradient for the top three input neurons for positive emotions (in d), negative emotions (in e), and both positive and negative emotions (in $\mathrm{f}$ ).

We also developed a Feature Interaction Network (FIN) that captures the level of spike interaction over time between the generated neural clusters of 14 EEG channels (brain regions) in terms of recognition of different emotional states. Fig. 6 ( $a$ and $b$ ) illustrates the neural clusters with dots and the level of internal spike interactions with lines.

The thicker the lines, the greater the spikes exchanged between the neural clusters during the STDP learning time. In both graphs of E1 and E2, most of the thicker lines were established in the left hemisphere, specifically between EEG channel T7 and channels FC5, F3, F7 and AF3, reflecting that more spikes were transmitted between the neurons placed in these sites of the brain. Additionally, there were broader connections between the neurons that were positioned in the parietal and posterior areas (O1 and P7 channels) in E1 and E2.

On the other hand, the EEG channels FC6, F4, F8, and AF4 which are in the right hemisphere, show stronger interactions in the SNN model of positive emotions (Fig. 6(a)) than the negative emotions (Fig. 6(b)). The findings revealed that positive and negative emotions have different cognitive processes in the brain and illustrate the areas of differences.

Each SNN model is supported by quantitative information that represents the number of spikes emitted in each neuron during the STDP learning process (shown in Fig. 6(c and d)). This measured the intensity of spikes produced by a neuron and sent to other neurons (post-spike); thus, information propagation occurs in the model.

\section{EEG Classification Using SNN Models}

The EEG datasets for different emotion states were learned in the SNN models using the unsupervised STDP learning. When the STDP learning is completed, the deSNN supervised learning technique was performed for classification of EEG samples to different emotions. At this stage, the relationship between the training EEG samples and their class label information is learned. The classification was performed using leave-one-out cross validation (LOOCV) with a gridsearch optimisation technique that optimised the main SNN's parameters with an objective function of reducing the classification error. Here, we selected three main parameters (STDP learning rate, mod and drift) to be optimised.

The parameters were selected by assigning 10 steps between the minimum and maximum values of each parameter range. Therefore, for every SNN model creation, 1000 iterations of training (using all samples except the holdout sample) and testing (using the single holdout sample) were performed with different combination of these three parameters. The classification accuracy between EEG samples of positive emotions (E1) versus negative emotions (E2) is reported in Table 1. The optimised parameters are STDP rate: 0.012 , the mod: 0.45 , and drift: 0.3 . Furthermore, the results of EEG data classification accuracy using SNN

TABLE 1: CLASSIFICATION BETWEEN EEG SAMPLES OF POSITIVE EMOTIONS (FROM E1) VERSUS NEGATIVE EMOTIONS (FROM E2) AND BETWEEN THE FOUR EMOTIONS (CALMNESS, HAPPINESS, FEAR, AND ANGER) USING BI-SNN MODELS AND TRADITIONAL METHODS.

\begin{tabular}{|c|c|c|c|c|c|}
\hline Session & Samples & NeuCube & MLR & MLP & RBF \\
\hline E3: 2 classes: positive emotions (E1) versus negative emotions (E2) & 92 (46 per class) & $\mathbf{8 3 . 5 \%}$ & $74.83 \%$ & $79.69 \%$ & $68.54 \%$ \\
\hline Experiment E4: 4 classes: calmness, happiness, fear, and anger & 92 (23 per class) & $94.83 \%$ & $78.88 \%$ & $72.96 \%$ & $74.06 \%$ \\
\hline
\end{tabular}


Positive Emotions (E1)

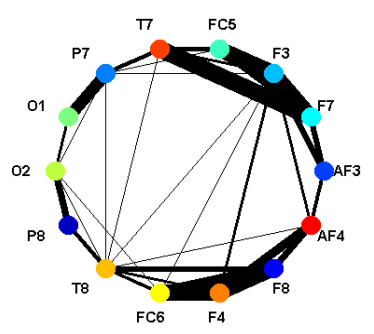

(a)

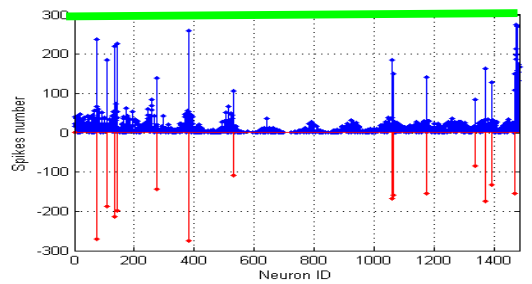

(c)
Negative Emotions (E2)

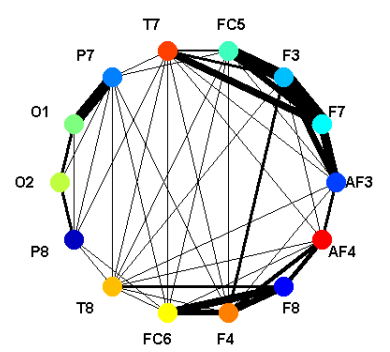

(b)

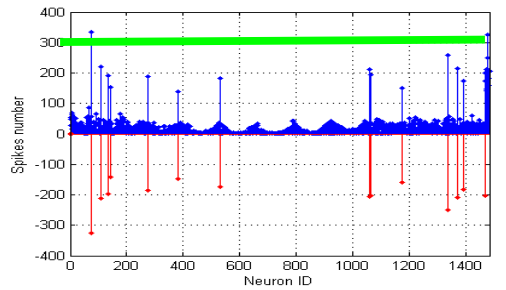

(d)

Fig. 6. Spike interaction between neural clusters of 14 EEG channels for positive emotions (a) and negative emotions (b). The number of positive and negative spikes produced by each neuron during the STDP learning in SNN models of positive emotions (c) and negative emotions (d).

models were compared with the outcomes of other machine learning methods such as Multi-Layer Perceptron (MLP), Radial Basis Function (RBF), and Multiple Linear Regression (MLR) obtained from NeuCom software v0.919 available at www.theneucom.com. The greatest classification accuracy of traditional methods was achieved by MLP of 79.69\%, which is less than the SNN model accuracy of $94.83 \%$. This shows that SNN models could differentiate the spatio-temporal patterns of EEG data much better when compared to other techniques (MLR, MLP, and RBF).

\section{CONCLUSION AND FUTURE DIRECTIONS}

This research explores the scope of emotion recognition by employing SNN architecture for modelling, mapping, learning, classifying, visualising and understanding of EEG brain data related to different emotional states. The obtained accuracy of $94.83 \%$ for EEG classification of four types of emotions was superior when compared with standard machine learning techniques (MLR, MLP, and RBF). The experimental study was divided into three sessions that consisted of 14 EEG channels from 23 healthy participants (14 Males, 9 Females). Experiment I focused on two classes of positive emotions (calmness, and happiness), Experiment II concentrated on two classes of negative emotions (fear, and anger), and finally Experiment III included all four emotions. A variety of techniques was implemented to analyse and classify the EEG data, including 3D visualisation of SNN connections (developed after unsupervised learning), spike emitted, neural clustering, feature interaction network, and max spike gradient. The research outcomes suggest there were differences between the SNN models of positive and negative emotions (from Experiment I and II) which were elaborated in this paper. Also, there is an increase in the connectivity of the frontal areas related to positive emotions, while the strength of connectivity was concentrated on the frontal and parietal sites of the brain for negative emotions. The classification accuracy in the SNN approach was higher when compared with the classification results using MLR, MLP, and RBF. As the future direction, the authors plan to use a larger dataset of EEG samples regarding emotional recognition. The next suggestion is to obtain new EEG datasets that involve older participants (age 50 or over) as a means of exploring emotional perception in different age groups. One practical application of this research is the creation of brain computer interface [4] towards developing human-computer interaction systems $[61,62]$.

\section{ACKNOWLEDGMENT}

The authors would like to acknowledge Maha Alzahrani, who helped with the formatting of the paper.

\section{REFERENCES}

[1] R. Khan and O. Sharif, "A literature review on emotion recognition using various methods," Global Journal of Computer Science and Technology, 2017.

[2] S. Katsigiannis and N. Ramzan, "DREAMER: A database for emotion recognition through EEG and ECG signals from wireless low-cost off-the-shelf devices," IEEE journal of biomedical and health informatics, vol. 22, no. 1, pp. 98-107, 2017.

[3] M. Soleymani and M. Pantic, "Multimedia implicit tagging using EEG signals," in 2013 IEEE International Conference on Multimedia and Expo (ICME), 2013: IEEE, pp. 1-6.

[4] A. Al-Nafjan, M. Hosny, Y. Al-Ohali, and A. Al-Wabil, "Review and classification of emotion recognition based on EEG braincomputer interface system research: a systematic review," Applied Sciences, vol. 7, no. 12, p. 1239, 2017.

[5] T. Pun, T. I. Alecu, G. Chanel, J. Kronegg, and S. Voloshynovskiy, "Brain-computer interaction research at the Computer Vision and Multimedia Laboratory, University of Geneva," IEEE Transactions on Neural Systems and Rehabilitation Engineering, vol. 14, no. 2, pp. 210-213, 2006.

[6] E. V. Friedrich et al., "An effective neurofeedback intervention to improve social interactions in children with autism spectrum disorder," Journal of autism and developmental disorders, vol. 45, no. 12, pp. 4084-4100, 2015.

[7] Y. Omori, H. Kawano, A. Seo, Z. G. Doborjeh, N. Kasabov, and M. G. Doborjeh, "EEG Comparison Between Normal and Developmental Disorder in Perception and Imitation of Facial Expressions with the NeuCube," in International Conference on Neural Information Processing, 2017: Springer, pp. 596-601. 
[8] S. Poria, E. Cambria, R. Bajpai, and A. Hussain, "A review of affective computing: From unimodal analysis to multimodal fusion," Information Fusion, vol. 37, pp. 98-125, 2017.

[9] G. Caridakis et al., "Multimodal emotion recognition from expressive faces, body gestures and speech," in IFIP International Conference on Artificial Intelligence Applications and Innovations, 2007: Springer, pp. 375-388.

[10] Y. Miyakoshi and S. Kato, "Facial emotion detection considering partial occlusion of face using Bayesian network," in 2011 IEEE Symposium on Computers \& Informatics, 2011: IEEE, pp. 96-101.

[11] H. K. Vydana, P. P. Kumar, K. S. R. Krishna, and A. K. Vuppala, "Improved emotion recognition using GMM-UBMs," in 2015 International Conference on Signal Processing and Communication Engineering Systems, 2015: IEEE, pp. 53-57.

[12] B. Schuller, G. Rigoll, and M. Lang, "Hidden Markov modelbased speech emotion recognition," in 2003 IEEE International Conference on Acoustics, Speech, and Signal Processing, 2003. Proceedings.(ICASSP'03). 2003, vol. 2: IEEE, pp. II-1.

[13] P. Mahendhiran and S. Kannimuthu, "Deep learning techniques for polarity classification in multimodal sentiment analysis," International Journal of Information Technology \& Decision Making, vol. 17, no. 03, pp. 883-910, 2018.

[14] S. Sun, C. Luo, and J. Chen, "A review of natural language processing techniques for opinion mining systems," Information fusion, vol. 36, pp. 10-25, 2017.

[15] F. Wang, S.-h. Zhong, J. Peng, J. Jiang, and Y. Liu, "Data augmentation for eeg-based emotion recognition with deep convolutional neural networks," in International Conference on Multimedia Modeling, 2018: Springer, pp. 82-93.

[16] E. S. Salama, R. A. El-Khoribi, M. E. Shoman, and M. A. W. Shalaby, "EEG-based emotion recognition using 3D convolutional neural networks," Int. J. Adv. Comput. Sci. Appl, vol. 9, no. 8, pp. 329-337, 2018

[17] E. Capecci, Z. G. Doborjeh, N. Mammone, F. La Foresta, F. C. Morabito, and N. Kasabov, "Longitudinal study of Alzheimer's disease degeneration through EEG data analysis with a NeuCube spiking neural network model," in 2016 International Joint Conference on Neural Networks (IJCNN), 2016: IEEE, pp. 13601366.

[18] E. Capecci, N. Kasabov, and G. Y. Wang, "Analysis of connectivity in NeuCube spiking neural network models trained on EEG data for the understanding of functional changes in the brain: A case study on opiate dependence treatment," Neural Networks, vol. 68, pp. 62-77, 2015.

[19] Z. Doborjeh et al., "Interpretability of Spatiotemporal Dynamics of the Brain Processes Followed by Mindfulness Intervention in a Brain-Inspired Spiking Neural Network Architecture," Sensors, vol. 20 , no. 24 , p. $7354,2020$.

[20] P. J. Sanders, Z. G. Doborjeh, M. G. Doborjeh, N. K. Kasabov, and G. D. Searchfield, "Prediction of Acoustic Residual Inhibition of Tinnitus Using a Brain-Inspired Spiking Neural Network Model," Brain Sciences, vol. 11, no. 1, p. 52, 2021.

[21] M. Durai et al., "Prediction of tinnitus masking benefit within a case series using a spiking neural network model," Progress in Brain Research, vol. 260, pp. 129-165, 2020.

[22] Y. Daşdemir, S. Yıldırım, and E. Yıldırım, "Classification of emotion primitives from EEG signals using visual and audio stimuli," in 2015 23nd Signal Processing and Communications Applications Conference (SIU), 2015: IEEE, pp. 2250-2253.

[23] M. A. Sarıkaya and G. İnce, "Emotion recognition from EEG signals through one electrode device," in 2017 25th Signal Processing and Communications Applications Conference (SIU), 2017: IEEE, pp. 1-4.

[24] A. Basu and A. Halder, "Facial expression and EEG signal based classification of emotion," in International Conference on Electronics, Communication and Instrumentation (ICECI), 2014: IEEE, pp. 1-4.

[25] C.-H. Han and C.-H. Im, "Data-driven user feedback: an improved neurofeedback strategy considering individual variability of EEG features," in The 18th IEEE International Symposium on Consumer Electronics (ISCE 2014), 2014: IEEE, pp. 1-2.
[26] M. Doborjeh, "Development of Spiking Neural Network Methods for Spatio-temporal Brain Data Analysis," Auckland University of Technology, 2019.

[27] M. G. Doborjeh and N. Kasabov, "Dynamic 3D clustering of spatio-temporal brain data in the NeuCube spiking neural network architecture on a case study of fMRI data," in International Conference on Neural Information Processing, 2015: Springer, pp. 191-198.

[28] N. Kasabov, L. Zhou, M. G. Doborjeh, Z. G. Doborjeh, and J. Yang, "New algorithms for encoding, learning and classification of fMRI data in a spiking neural network architecture: A case on modeling and understanding of dynamic cognitive processes," IEEE Transactions on Cognitive and Developmental Systems, vol. 9, no. 4, pp. 293-303, 2016.

[29] N. K. Kasabov, M. G. Doborjeh, and Z. G. Doborjeh, "Mapping, learning, visualization, classification, and understanding of fMRI data in the NeuCube evolving spatiotemporal data machine of spiking neural networks," IEEE transactions on neural networks and learning systems, vol. 28, no. 4, pp. 887-899, 2016.

[30] M. G. Doborjeh, E. Capecci, and N. Kasabov, "Classification and segmentation of fMRI spatio-temporal brain data with a NeuCube evolving spiking neural network model," in 2014 IEEE Symposium on Evolving and Autonomous Learning Systems (EALS), 2014: IEEE, pp. 73-80.

[31] N. K. Kasabov, "NeuCube: A spiking neural network architecture for mapping, learning and understanding of spatio-temporal brain data," Neural Networks, vol. 52, pp. 62-76, 2014.

[32] N. Kasabov, "Neucube evospike architecture for spatio-temporal modelling and pattern recognition of brain signals," in Iapr workshop on artificial neural networks in pattern recognition, 2012: Springer, pp. 225-243.

[33] N. Kasabov et al., "Evolving spatio-temporal data machines based on the NeuCube neuromorphic framework: Design methodology and selected applications," Neural Networks, vol. 78, pp. 1-14, 2016.

[34] N. K. Kasabov, Time-space, spiking neural networks and braininspired artificial intelligence. Springer, 2019.

[35] S. Budhraja, B. S. Bhattacharya, S. Durrant, Z. Doborjeh, M. Doborjeh, and N. Kasabov, "Sleep Stage Classification using NeuCube on SpiNNaker: a Preliminary Study," in 2020 International Joint Conference on Neural Networks (IJCNN), 2020: IEEE, pp. 1-8.

[36] M. Doborjeh, N. Kasabov, Z. Doborjeh, R. Enayatollahi, E. Tu, and A. H. Gandomi, "Personalised modelling with spiking neural networks integrating temporal and static information," Neural Networks, vol. 119, pp. 162-177, 2019.

[37] M. G. Doborjeh et al., "From von Neumann Architecture and Atanasoff's ABC to Neuromorphic Computation and Kasabov's NeuCube. Part II: Applications," in Practical Issues of Intelligent Innovations: Springer, 2018, pp. 17-36.

[38] M. G. Doborjeh, N. Kasabov, and Z. G. Doborjeh, "Evolving, dynamic clustering of spatio/spectro-temporal data in 3D spiking neural network models and a case study on EEG data," Evolving systems, vol. 9, no. 3, pp. 195-211, 2018.

[39] M. G. Doborjeh, G. Y. Wang, N. K. Kasabov, R. Kydd, and B. Russell, "A spiking neural network methodology and system for learning and comparative analysis of EEG data from healthy versus addiction treated versus addiction not treated subjects," IEEE transactions on biomedical engineering, vol. 63, no. 9, pp. 1830-1841, 2015.

[40] Z. Doborjeh et al., "Spiking neural network modelling approach reveals how mindfulness training rewires the brain," Scientific reports, vol. 9, no. 1, pp. 1-15, 2019.

[41] Z. G. Doborjeh, M. Doborjeh, and N. Kasabov, "EEG Pattern Recognition using Brain-Inspired Spiking Neural Networks for Modelling Human Decision Processes," in 2018 International Joint Conference on Neural Networks (IJCNN), 2018: IEEE, pp. $1-7$.

[42] Z. G. Doborjeh, M. G. Doborjeh, and N. Kasabov, "Attentional bias pattern recognition in spiking neural networks from spatiotemporal EEG data," Cognitive Computation, vol. 10, no. 1, pp. 35-48, 2018.

[43] Z. G. Doborjeh, N. Kasabov, M. G. Doborjeh, and A. Sumich, "Modelling peri-perceptual brain processes in a deep learning 
spiking neural network architecture," Scientific reports, vol. 8, no. 1, pp. 1-13, 2018.

[44] Z. Gholami Doborjeh, "Modelling of Spatiotemporal EEG and ERP Brain Data for Dynamic Pattern Recognition and Brain State Prediction using Spiking Neural Networks: Methods and Applications in Psychology," Auckland University of Technology, 2019.

[45] H. Kawano, A. Seo, Z. G. Doborjeh, N. Kasabov, and M. G. Doborjeh, "Analysis of similarity and differences in brain activities between perception and production of facial expressions using EEG data and the NeuCube spiking neural network architecture," in International conference on neural information processing, 2016: Springer, pp. 221-227.

[46] D. Shah, G. Y. Wang, M. Doborjeh, Z. Doborjeh, and N. Kasabov, "Deep learning of eeg data in the neucube braininspired spiking neural network architecture for a better understanding of depression," in International Conference on Neural Information Processing, 2019: Springer, pp. 195-206.

[47] A. Tavanaei, M. Ghodrati, S. R. Kheradpisheh, T. Masquelier, and A. Maida, "Deep learning in spiking neural networks," Neural Networks, vol. 111, pp. 47-63, 2019.

[48] X. Wu, V. Saxena, K. Zhu, and S. Balagopal, "A CMOS spiking neuron for brain-inspired neural networks with resistive synapses and in situ learning," IEEE Transactions on Circuits and Systems II: Express Briefs, vol. 62, no. 11, pp. 1088-1092, 2015.

[49] W. Maass, "Networks of spiking neurons: the third generation of neural network models," Neural networks, vol. 10, no. 9, pp. 1659-1671, 1997.

[50] S. Song, K. D. Miller, and L. F. Abbott, "Competitive Hebbian learning through spike-timing-dependent synaptic plasticity," Nature neuroscience, vol. 3, no. 9, pp. 919-926, 2000.

[51] E. Tu et al., "Neucube (st) for spatio-temporal data predictive modelling with a case study on ecological data," in 2014 international joint conference on neural networks (IJCNN), 2014: IEEE, pp. 638-645.

[52] W. Maass, T. Natschläger, and H. Markram, "Real-time computing without stable states: A new framework for neural computation based on perturbations," Neural computation, vol. 14 , no. 11 , pp. $2531-2560,2002$

[53] J. Talairach, "3-dimensional proportional system; an approach to cerebral imaging. co-planar stereotaxic atlas of the human brain," Thieme, pp. 1-122, 1988.

[54] V. Chan, S.-C. Liu, and A. van Schaik, "AER EAR: A matched silicon cochlea pair with address event representation interface," IEEE Transactions on Circuits and Systems I: Regular Papers, vol. 54, no. 1, pp. 48-59, 2007.

[55] M. Brett, K. Christoff, R. Cusack, and J. Lancaster, "Using the Talairach atlas with the MNI template," Neuroimage, vol. 13, no. 6, pp. 85-85, 2001.

[56] D. J. Watts and S. H. Strogatz, "Collective dynamics of 'smallworld'networks," nature, vol. 393, no. 6684, pp. 440-442, 1998.

[57] N. Kasabov, K. Dhoble, N. Nuntalid, and G. Indiveri, "Dynamic evolving spiking neural networks for on-line spatio-and spectrotemporal pattern recognition," Neural Networks, vol. 41, pp. 188201, 2013.

[58] M. Claesen and B. De Moor, "Hyperparameter search in machine learning," arXiv preprint arXiv:1502.02127, 2015.

[59] C. A. Gabert-Quillen, E. E. Bartolini, B. T. Abravanel, and C. A. Sanislow, "Ratings for emotion film clips," Behavior research methods, vol. 47, no. 3, pp. 773-787, 2015.

[60] D. H. Johnson, "Signal-to-noise ratio," Scholarpedia, vol. 1, no. 12, p. 2088, 2006.

[61] C. Tan, G. Ceballos, N. Kasabov, and N. Puthanmadam Subramaniyam, "FusionSense: Emotion Classification Using Feature Fusion of Multimodal Data and Deep Learning in a Brain-Inspired Spiking Neural Network," Sensors, vol. 20, no. 18, p. 5328, 2020.

[62] C. Tan, M. Šarlija, and N. Kasabov, "NeuroSense: Short-term emotion recognition and understanding based on spiking neural network modelling of spatio-temporal EEG patterns," Neurocomputing, vol. 434, pp. 137-148, 2021. 